This is an author produced version of a paper published in Archives of Orthopaedic and Trauma Surgery. This paper has been peer-reviewed but does not include the final publisher proof-corrections or journal pagination.

Citation for the published paper:

W-Dahl, Annette and Toksvig-Larsen, Soren.

"Infection prophylaxis: a prospective study in 106 patients operated on by tibial osteotomy using the hemicallotasis technique."

Archives of Orthopaedic and Trauma Surgery, 2006, Vol: 126, Issue: 7, pp. 441-7.

http://dx.doi.org/10.1007/s00402-006-0165-y

Access to the published version may require journal subscription. Published with permission from: Springer 


\title{
Infection prophylaxis
}

\section{A prospective study in $\mathbf{1 0 6}$ patients operated on by tibial osteotomy using the hemicallotasis technique}

\author{
Annette W-Dahl, Sören Toksvig-Larsen \\ Department of Orthopedics, University Hospital, Lund, Sweden
}

Correspondence to:

Annette W-Dahl

Department of Orthopedics

University Hospital

S-221 85 Lund

Sweden

Phone +4646175350

Fax +4646146487

annette.w-dahl@med.lu.se 


\begin{abstract}
Introduction Tibial osteotomy by the hemicallotasis technique is a clean elective operation. With external fixation pins inserted, close to the knee joint, the infection prophylaxis should be considered. The primary aim was to investigate the differences in the postoperative use of antibiotics during the time in external fixation between administrating prophylactic antibiotics for 3 days or as a single dose in patients operated on by the hemicallotasis technique for knee deformities. Secondary aims were to study the differences in pin-site infection rate and grade and complications.

Material and methods A total of 106 consecutive patients of mean age 52 years (range 18-69) operated on by the hemicallotasis technique for knee deformities were included in this prospective study. Sixty patients were prescribed prophylactic antibiotics for 3 days and 46 patients as a single dose. Chlorhexidine $(5 \mathrm{mg} / \mathrm{ml})$ in alcohol (70\% ethanol) was used as cleansing agent in the pin-site care. The power of the study was calculated to $80 \%$ to detect a difference in the postoperative use of antibiotics for 7 days during the treatment in external fixation.

Results There were no differences in postoperative use of antibiotics between 3 days administration or a single dose of prophylactic antibiotics. This was the case with infection rate and grade, positive bacterial cultures, presence of Staphylococcus aureus, nor positive culturing from the tip of the pins at removal. Neither were there any differences in numbers of loose pins and complications.

Conclusion There were no differences between 3 days of administration of prophylactic antibiotics and one single dose. One single dose of prophylactic antibiotics is appropriate together with a pin-site concept preventing pin-site infection in patients operated on by hemicallotasis osteotomy.
\end{abstract}

Keywords Antibiotic prophylaxis · Infection control · External Wxators · Tibial osteotomy • Chlorhexidine

\title{
Introduction
}

The optimal duration of prophylactic antibiotics is unknown but reports have documented effective prophylaxis with a single dose in orthopedic joint-replacement surgery [1-4]. However, the use of prophylaxis for longer periods has been advocated [5-7]. Tibial osteotomy by the hemicallotasis technique is a clean elective surgery. With external fixation pins inserted, close to the knee joint, the infection prophylaxis should be considered. The risk for a severe infection is low, but the consequences can be serious. The development of bacterial resistance is associated with antimicrobial use. Prophylactic antibiotics should be used as little as possible and the spectrum of activity of drugs used should be as narrow as possible [8].

During several years, we have documented pin-site infection and in an attempt to decrease the infection problems and the use of antibiotics during the treatment by hemicallotasis osteotomy (HCO) $[9,10]$, we changed the pin-site care step by step together with the use of infection prophylaxis. The aim of the present study was to investigate the diVerences in pin-site infection and other complications between administrating the prophylactic antibiotics for 3 days or as a single dose in patients operated on by the hemicallotasis technique for knee deformities.

\section{Method}

Patients

A total of 106 patients (64 men and 42 women) mean age 52 (range 18-69) years operated on by the hemicallotasis technique for knee deformities (Table 1) were included consecutively. The 
patients were operated on consecutively, group 1 during the year 2000 and group 2 during 2001 . The 60 patients, ( 27 women) in group 1 , were given prophylactic antibiotics as one dose intravenous (Cloxacillin 2 g) before surgery, followed by two intravenous doses during the Wrst $24 \mathrm{~h}$ followed by per oral prophylaxis (Flucloxacillin $1 \mathrm{~g} £ 3$ ) for additional $48 \mathrm{~h}$. If the patient were discharged within $24 \mathrm{~h}$, the last intravenous dose was changed to oral dose. The 46 patients in group 2 (15 women) were given prophylactic antibiotics as a single intravenous dose (Cloxacillin $2 \mathrm{~g}$ ) before surgery. In the case of known allergic reaction to penicillin, the patient was given Clindamycin $300 \mathrm{mg}$. The local recommendation was to administrate the preoperative prophylactic antibiotics 20-30 min before the incision. Preoperative antiseptic showering DesCutan ${ }^{\circledR} 4 \%$ (Chlorhexidine gluconate 4 g) were done twice, the first in the evening before surgery and the second in the morning the day for the surgery.

\section{Hemicallotasis osteotomy}

Four conical pins were inserted, two hydroxyapatite (HA) coated in the metaphyseal bone and two standard pins (Orthofix ${ }^{\circledR}$, Bussolengo, Italy) in the diaphyseal bone. The Orthofix ${ }^{\circledR} \mathrm{T}$ garche was used as the external fixator. A longitudinal skin incision, ventral to the tibial tuberosity, was done for the osteotomy. The operation was done without bloodless field. Sterile compresses moistened by Chlorhexidine $(5 \mathrm{mg} / \mathrm{ml}$ ) in alcohol (ethanol 70\%) were draped around each pin site and an absorbent pad covered the incision and fixed by a bandage were used as the operative dressing. The dressing was in situ until the first outpatient visit 1 week postoperatively. Patients were allowed to do free mobilization and full weight bearing postoperatively. Fifty-five percent (58/106) of the patients was discharged at the same day as surgery. The distraction starts 7 to 10 days postoperatively. Eight weeks postoperation, the fixation was dynamized to stimulate the bone healing. At 12 weeks postoperation, a bone-healing control was done by radiographic and ultrasound investigation. If the osteotomy healing was satisfying, the patient did a weight-bearing test, i.e., walking an extended period of time varying from some hours to some days without the instrument but still with the pins in situ. If no symptoms arise, the pins were removed in the out patient clinic. If the patient developed symptoms, the fixator was applied for additional 2-3 weeks.

\section{Pin-site care}

A nurse performed the pin-site care once a week in the orthopedic outpatient clinic. The clean technique (sterile material and clean gloves) was used in the orthopaedic outpatient clinic. All bandages were removed. Each pin site was cleaned by chlorhexidine alcohol ( $5 \mathrm{mg} / \mathrm{ml})$ in alcohol (ethanol 70\%). No crusts were removed unless signs of infection perceived. A sterile compress moistened with Chlorhexidine (5 mg/ml) in alcohol (ethanol 70\%) was placed at each pin site and was fixed by a soft dressing around each pair of pins. When showering, the patient protected the pin sites using a plastic bag. The patients had full access to the out patient clinic if they had questions or any problems occurred. In the case of pin-site infection or drainage, extra visits were made if needed.

Antibiotic treatment 
Flucloxacillin $1 \mathrm{~g} £ 3$ or the antimicrobial drug susceptible for the positive culture was used for 7 days as antibiotic treatment during the treatment period.

\section{Outcome}

The postoperative use of antibiotics was recorded for a number of days and used as the primary outcome of presence of pin-site infection during the treatment in external fixation. Pin sites were clinically classified according to the Checketts-Otterburns classification

[11], bacterial culturing was taken from each pin site at the first, sixth and tenth week and from the tip of the pins at removal and complications were recorded. At removal, the pins were assessed as clinically loose or fixed. A loose pin was defined as a pin, which could be removed by hand without use of a wrench. The time for administration of the preoperatively prescribed prophylactic antibiotics was controlled retroperspectively by analyzing the medical records. The time of the preoperative antibiotic dose given was classified in patients administrated the preoperative dose $\cdot 60$ min before the first incision and patients administrated the preoperative dose at any other occasion or not at all. The extent of antibiotic treatment clinical infection graded according to the Checketts-Otterburns classification [11], number of positive cultures, the presences of Staphylococcus aureus and the number of loose pins at extraction were compiled as well as the complications. Grade I-III according to the Checketts-Otterburns classification [11] was classified as minor infections and grade IV-VI as major infections. Complications included delayed healing ( $>112$ days in external fixation), pseudoarthrosis, septic arthritis, deep venous thrombosis, nerve damage and interrupted treatment. The patients were defined as non-smokers if they, at the preoperative visit, reported that they never had smoked or stopped smoking since more than 6 months. Frame time (from surgery until the external pins were removed) was documented.

\section{Statistic analysis}

The analysis of variance (ANOVA) test, and Chisquare test or Fisher's exact test were used for the statistical analysis, statistical level $P<0.05$. A multiple logistic regression analysis (controlled for potential confounders) was used to estimate the odds ratio (OR) of positive bacterial cultures and presence of $S$. aureus at the pin sites at any occasion during the treatment and other complications. Starting with the univariate model and selected variables that had a $P$ value $<0.25$ was considered as candidates for the multivariate models [12]. We entered the variables into multivariate models if they changed the eVect estimated by $10 \%$ or more, and we excluded them if the exclusion changed the estimate by less than 5\% [13]. As potential confounders gender, age, body mass index (BMI), prophylactic antibiotics, hospital stay, smoking habits and administration time of the prophylactic antibiotics were entered in the multiple logistic regression model. The power of the study was calculated to $80 \%$ to detect a difference in the postoperative use of antibiotics for 7 days during the treatment in external fixation; 33 patents in each group were needed. The study was approved by the Ethics Committee, Lund University, Sweden.

\section{Result}

Antibiotics 
The total mean antibiotic consumption per patient (prophylaxis plus antibiotic treatment) during the treatment in external fixation was 13 days (SD 14) for group 1 and

11 days (SD 11) for group 2. The mean difference was 2 days (95\% CI $; 3.0-7.0, P=0.4$ ). There were no differences in the number of patients treated by the antibiotic: 30/60 patients in group 1 and 32/46 patients in group 2, RR 1.4 (95\% CI 1.0-1.9, $P=0.07)$. Neither were there any difference in the mean antibiotic treatment per treated patient (prophylaxis excluded): 15 days (SD 12) in group 1 and 17 days (SD 10) in group 2, the mean difference were 2 days (95\% CI i7.6-3.6, $P=0.5)$.

Infection

There were no differences in the postoperative use of antibiotics during the treatment in external fixation administrated 3 days or one single dose of prophylactic antibiotics. There were no differences for positive bacterial cultures or presence of $S$. aureus at the pin sites at week 1, 6 and 10 or at any time during the treatment by HCO (Table 2). Neither were there any differences for bacterial cultures from the pin tips at extraction (Table 3). There were no differences in the clinical assessment according to the Checketts-Otterburns classification (Table 2). There were no major infections. When adjusted for potential risk factors, patients who stayed for 1 day at the hospital [,2 days OR 0.2 (95\% CI 0.08- 0.8, $P=0.02$ ] and non-smokers [smokers OR 0.2 (95\% CI $0.05-0.8), P=0.02]$ were the risk factors for the presence of $S$. aureus during the treatment by HCO (Table 4).

\section{Complications}

There were no differences in the number of patients with loose pins at extraction, positive bacterial cultures and presence of $S$. aureus in positive bacterial cultures at the pin tip at extraction (Table 3). The total number of loose pins at extraction was 14/256 in the group 1 and $11 / 200$ group 2 [RR 0.5 (95\%CI $0.2-1.1), P=0.1$ ]. Neither were there any differences in the number of patients with complications 14/60 in group 1 and 12/46 in group 2, [RR 1.1 (95\% CI 0.5-2.3), $P=0.9$ ] or in patients with more than one complication $4 / 60$ in group 1 and 2/46 in group 2, [RR 0.6 (95\% CI 0.08-3.9), $P=0.9$ ]. When the results were adjusted for risk factors, the hospital stay for more than 1 day and smokers were found to be risk factors for complications during the treatment by HCO (Table 4). The treatment was interrupted in one patient in group 1 due to pin-site infection (Citrobacter diversus in the two proximal pins and in one of the distal pins) and loose pins at week 6 (S. aureus at one proximal and one distal pin). The correction and healing was obtained by additional surgery. Two patients developed pseudoarthrosis (one patient with osteonecrosis after the treatment for leukaemia and one smoker). Both healed after additional surgery. One patient in the group 2 developed a septic arthritis (Staphylococcus lugdunensis) after 12 weeks in external fixation and has been converted to a total knee arthroplasty.

Timing of administrated prophylactic antibiotics 
In group 1, 16/60 and group 2, 2/46 patients were given the prescribed preoperative dose of antibiotics postoperatively, and 10/60 (group 1) and 5/46 (group 2) patients were not given any preoperative prophylactic antibiotics at all according to the medical records. There was a significant difference between the two groups regarding when the preoperative antibiotic dose was given. In group 1, 34/60 patients got the preoperative dose of antibiotics 60 min preoperatively and 39/46 in group $2(P=0.002)$. However, there were no differences in numbers of positive bacterial cultures, presence of $S$. aureus or numbers of positive culturing from the pin tip at extraction. Neither were there any differences in number of loose pins, complications or the use of antibiotics between patients who had been given the preoperative dose of antibiotics $\cdot 60$ min before surgery or at any other occasion.

\section{Discussion}

There were no differences in the results using infection prophylaxis by antibiotics for 3 days or a single dose preoperatively in patients operated on by the hemicallotasis technique. Unexpected findings in the multivariate logistic regression analysis were that patients who stayed only 1 day at the hospital and non-smokers were the risk factors for the presence of S. aureus. The risk factors for the presence of $S$. aureus would be expected to be the opposite. Longer time of stay in the hospital environment ought to be a higher risk of infection. Reviewing the literature, no studies were found showing that a short hospital stay should be a benefit for minor wound infections. Several studies showed that a shorter preoperative stay was a benefit $[14,15]$ and patients with postoperative infections and complications had a prolonged hospital stay $[15,16]$. Smoking has been shown to be a risk factor of wound infection [17-19]. The multivariate logistic regression analysis showed that patients, who stayed for more than 1 day in the hospital and smokers were the risk factors for developing complications. An optimal infection prophylaxis includes an antimicrobial drug, with a broad spectrum, covering the organisms most likely to cause infection and achieve adequate concentration before the first incision and maintain a sufficient concentration until closure of the skin $[8,20]$. The timing of prophylaxis by antibiotics is important to get a high antibiotic concentration at the time for skin incision. This is considered to be optimal about $30 \mathrm{~min}$ before skin incision, i.e. at the induction of anesthesia $[8,20]$. As the optimal timing of prophylactic antibiotics is unknown and different suggestions are made just before the incision [2], for instance, antibiotics given longer than 60 min preoperatively were associated with a higher rate of infectious complications [21]. Therefore, it was chosen in this study to divide the patients into a group that were given the preoperative prophylaxis $\cdot 60$ min preoperatively and a second group which got the prophylactic antibiotics at any other time or not at all. The intension of this study was to administrate for 3 days, one dose of prophylactic antibiotics and this prescription was given in the medical records. When we retroperspectively controlled the medical records, $31 \%$ of the patients were given the prescribed prophylaxis postoperatively or not at all. Despite the fact that these patients did not follow the study protocol, we let them stay included in the study and controlled for the time of prophylactic antibiotics given when using the regression analysis. This raised the question if prophylaxis is necessary in patients operated on by HCO, but it also questions the effectiveness in the surgical organization. Three doses [22] as well as 1 [23] to 2 weeks [7] of antibiotics has been reported as infection prophylaxis for the hemicallotasis technique. Magyar et al. [7] found that the overall relative risk for pin-site infection decreased with prolonged prophylactic antibiotics up to 10-14 days. The effect of different infection prophylaxis by antibiotics performing HCO is diffcult to evaluate when there are various definitions of infection. The results of this present study with 6 and $7 \%$ of 
minor pin-site infection according to the Checketts- Otterburns classification using 3 days each of one dose of prophylactic antibiotics indicated that prolonged postoperative use of antibiotics has no benefit. One single dose of Cloxacillin $2 \mathrm{~g}$ intravenous 20-30 min before the Wrst incision is the local recommendation as infection prophylaxis by antibiotics in surgery for more than $1 \mathrm{~h}$ in our department. The time of surgery of HCO is about $30 \mathrm{~min}$. The half-life of Cloxacillin is relatively short (30 min in serum) but Cloxacillin reach a high concentration in serum proteins (94\%) and has an appropriate spectrum of coverage of the most likely pathogens associated with pin-site infections. As $S$. aureus is the organism predominantly causing incision surgical infections [8, 20, 24] and pin-site infections [11], we used Chlorhexidine (5 mg/ml) in alcohol (70\% ethanol) as the cleansing agent. Chlorhexidine has the advantages of a low toxicity, broad spectra of antimicrobial activity. Chlorhexidine together with alcohol reduces the skin micro Xora and has a residual antimicrobial activity [25]. A clinical difference of 7 days was used to detect a difference between the two doses of prophylactic antibiotics in the power calculation. Seven days represented one cure of treatment in the case of pin-site infection. The choice of use of antibiotics during the treatment in external fixation as primary outcome of the study was done by the reason that a low use of antibiotics indicates a low pin-site infection rate. We conclude that one single dose of antibiotic prophylaxis is appropriate together with a pin-site concept preventing pin-site infection during the treatment of HCO.

\section{References}

1. Mazza A (2000) Ceftriaxone as short-term antibiotic prophylaxis in orthopedic surgery: a costbenefit analysis involving 808 patients. J Chemother 12(Suppl 3):29-33

2. Barie PS (2000) Modern surgical antibiotic prophylaxis and therapy-less is more. Surg Infect (Larchmt) 1:23-29

3. Esposito S (1999) Is single-dose antibiotic prophylaxis sufficient for any surgical procedure? J Chemother 11:556-564 Arch Orthop Trauma Surg (2006) 126:441-447 447123

4. Schmidt-Matthiesen A, Roding H, Windolf J, Sommerfeldt D, Pannike A, Encke Al (1999) A prospective, randomised comparison of single- vs. multiple-dose antibiotic prophylaxis in penetrating trauma. Chemotherapy 45:380-391

5. Wittmann DH, Schein M (1996) Let us shorten antibiotic prophylaxis and therapy in surgery. Am J Surg 172:26S-32S

6. Strachan CJ (1993) Antibiotic prophylaxis in peripheral vascularand orthopaedic prosthetic surgery. J Antimicrob Chemother 31(Suppl B):65-78

7. Magyar G, Ahl TL, Vibe P, Toksvig-Larsen S, Lindstrand A (1999) Open-wedge osteotomy by hemicallotasis or the closed-wedge technique for osteoarthritis of the knee: a randomised study of 50 operations. J Bone Joint Surg Br 81:444-448

8. Gyssens IC (1999) Preventing postoperative infections: current treatment recommendations. Drugs 57:175-185

9. W-Dahl A, Toksvig-Larsen S, Lindstrand A (2003) No differgence between daily and weekly pin site care: a randomized study of 50 patients with external fixation. Acta Orthop Scand 74:704-708

10. W-Dahl A, Toksvig-Larsen S (2004) Pin site care in external fixation sodium chloride or chlorhexidine solution as a cleansing agent. Arch Orthop Trauma Surg 124:555-558

11. Checketts RG, Moran CG, Mac Eachern AG, Otterburn M (1999) Pin track infecktion and the principles of pin site care. In: De Bastiani G, Graham Apley A, Goldberg A (eds) OrthoWx 
external Wxation in trauma and orthopeadics. Springer, London Berlin Heidelberg, pp 97-103

12. Hosmer D, Lemeshow S. (1989) Applied logistic regression. Wiley, New York

13. Greenland S. (1994) Modeling and variable selection in epidemiologic analysis. Am J Public Health 79:340-349

14. Wilson AP, Livesey SA, Treasure T, Gruneberg RN, Sturridge MF (1987) Factors

predisposing to wound infection in cardiac surgery: a prospective study of 517 patients. Eur $\mathrm{J}$

Cardiothorac Surg 1:158-164

15. Anielski R, Barczynski M (1998) Postoperative wound infections. I. Population data and risk factors. Przegl Lek 55:101-108

16. Smilanich RP, Bonnet I, Kirkpatrick JR (1995) Contaminated wounds: the eVect of initial management on outcome. Am Surg 61:427-430

17. Moller AM, Pedersen T, Villebro N, Munksgaard A (2003) Effect of smoking on early complications after elective orthopaedic surgery. J Bone Joint Surg (Br) 85-B:178-181

18. Moller AM, Villebro N, Pedersen T, Tonnesen H (2002) Effect of preoperative smoking intervention on postoperative complications: a randomised clinical trial. Lancet 359:114-117 19. W-Dahl A, Toksvig-Larsen S (2004) Cigarett smoking delays bone healing: a prospective study of 200 patients operated on by the hemicallotasis technique. Acta Orthop Scand 75:347351

20. Polk HC Jr, Christmas AB (2000). Prophylactic antibiotics in surgery and surgical wound infections. Am Surg 66:105-111

21. Galandiuk S, Polk HC Jr, Jagelman DG, Fazio VW (1989) Re-emphasis of priorities in surgical antibiotic prophylaxis. Surg Gynecol Obstet 169:219-222

22. Weale AE, Lee AS, MacEachern AG (2001) High tibial osteotomy using a dynamic axial external fixator. Clin Orthop 154-167

23. Magyar G, Toksvig-Larsen S, Lindstrand A (1998) Open wedge tibial osteotomy by callus distraction in gonarthrosis: operative technique and early results in 36 patients. Acta Orthop Scand 69:147-151

24. Mangram AJ, Horan TC, Pearson ML, Silver LC, Jarvis WR (1999) Guideline for prevention of surgical site infection, 1999. Hospital Infection Control Practices Advisory Committee. Infect Control Hosp Epidemiol 20:250-278 (quiz 279-280)

25. Nakamura E, Mizuta H, Kudo S, Takagi K, Sakamoto K (2001) Open-wedge osteotomy of the proximal tibia hemicallotasis. J Bone Joint Surg Br 83:1111-1115 
Table 1. Patient characteristics of the study group.

\begin{tabular}{|c|c|c|c|}
\hline & $\begin{array}{l}\text { All } \\
\mathrm{N}=106\end{array}$ & $\begin{array}{l}3 \text { days } \\
\text { prophylaxis } \\
n=60\end{array}$ & $\begin{array}{l}\text { One dose } \\
\text { prophylaxis } \\
\mathrm{n}=46\end{array}$ \\
\hline \multicolumn{4}{|l|}{ Gender (n) } \\
\hline Men & 64 & 31 & 33 \\
\hline Women & 42 & 27 & 15 \\
\hline Age mean (years) & 52 & 52 & 52 \\
\hline SD & 9 & 11 & 7 \\
\hline \multicolumn{4}{|c|}{ Antibiotic prophylaxis (n) } \\
\hline Cloxacillin & 103 & 58 & 45 \\
\hline Clindamycin & 3 & 2 & 1 \\
\hline \multicolumn{4}{|l|}{$\begin{array}{l}\text { Indication of operation } \\
\text { (number of knees) }\end{array}$} \\
\hline OA medial & 92 & 48 & 44 \\
\hline OA lateral & 13 & 10 & 3 \\
\hline Other* medial & 3 & 2 & 1 \\
\hline Other* lateral & 7 & 5 & 2 \\
\hline $\begin{array}{l}\text { Bilateral (n) } \\
\text { (simultaneous surgery) }\end{array}$ & 9 & 5 & 4 \\
\hline Frame time (days) & 99 & 97 & 102 \\
\hline SD & 18 & 19 & 18 \\
\hline \multicolumn{4}{|c|}{ Pre HKA-angle (degrees) } \\
\hline Mean medial (lateral) & $170(187)$ & $170(188)$ & $170(185)$ \\
\hline SD medial (lateral) & $5(6)$ & $5(6)$ & $5(5)$ \\
\hline
\end{tabular}


$\begin{array}{llll}\text { Mean } & 25 & 24 & 27\end{array}$

$\begin{array}{llll}\text { SD } & 11 & 10 & 12\end{array}$

$\begin{array}{llll}\mathrm{BMI}\left(\mathrm{kg} / \mathrm{m}^{2}\right) & 29 & 28 & 30\end{array}$

$\begin{array}{llll}\mathrm{SD} & 5 & 5 & 4\end{array}$

$\begin{array}{llll}\text { Smoker (n) } & 22 & 14 & 8\end{array}$

$\begin{array}{llll}\text { Diabetes (n) } & 1 & 0 & 1\end{array}$

(tablet treatment)

* Sequel fracture, correction and osteonecrosis.

$\mathrm{SD}=$ Standard deviation, Inf prophylaxis= Infection prophylaxis by antibiotics, $\mathrm{OA}=$

osteoarthritis, Pre-HKA = radiographic Hip-Knee-Ankle angle, BMI = Body mass index 
Table 2. Positive bacteria cultures, presence of Staphylococcus aureus in positive bacteria cultures and clinical pin site infection according to Checketts-Otterburns classification ${ }^{4}$ of 106 patients operated on by the HCO.

Group 1 Group 2

Bacteria culture, Bacteria culture, clinical infection/ clinical infection/ RR (95\% CI) p value patient patient Group 1 $\mathrm{n}=60 \quad \mathrm{n}=46$ 1.0 (Ref)

Week 1

Pos bact cult $21 / 60$

$11 / 44$

0.7 (0.4-1.4)

ns

Staph aureus*

$4 / 60$

$3 / 44$

$1.0(0.2-5.2)$

ns

Grade 1

$2 / 60$

$0 / 46$

Grade 2

$0 / 60$

$0 / 46$

Week 6

Pos bact cult

$18 / 52$

$11 / 52$

17/36

$13 / 36$

$8 / 55$

$9 / 42$

3/55

2/42

$1.4(0.8-2.4)$

$1.7(0.8-3.7)$

$0.7(0.3-1.8)$

$1.1(0.2-9.7)$

ns

ns

Grade 1

Grade 2

Week 10

Pos bact cult

$\begin{array}{ll}19 / 52 & 15 / 42 \\ 6 / 52 & 11 / 42 \\ 10 / 56 & 7 / 42 \\ 2 / 56 & 3 / 42\end{array}$

$1.0(0.5-1.8)$

ns

Staph aureus*

$11 / 42$

Grade 1

2/56

$3 / 42$

$2.2(0.8-6.5)$

ns

$1.0(0.4-2.9)$

ns

Grade 2

At any occasion**

Pos bact cult

\begin{tabular}{ll}
$41 / 60$ & $25 / 45$ \\
$18 / 60$ & $25 / 45$ \\
$17 / 60$ & $20 / 46$ \\
$6 / 60$ & $5 / 46$ \\
\hline
\end{tabular}

$0.5(0.06-3.5)$

ns

Staph aureus*

Grade 1

$6 / 60$

$5 / 46$

$0.8(0.6-1.2)$

ns

$1.3(0.7-2.4) \quad$ ns

Grade 2

$0.7(0.4-1.2)$

ns

* Presence of Staphylococcus aureus in positive bacteria cultures

** A positive bacteria culture/presence of Staphylococcus aureus in positive bacteria cultures/clinical pin site infection at week one and/or week 6 and/or week10

Group 1 = 3 days of infection prophylaxis by antibiotics, Group 2 = one dose of infection prophylaxis by antibiotics.

$\mathrm{HCO}=$ hemicallotasis osteotomy, Pos bact cult = positive bacteria cultures, $\mathrm{RR}=$ relative risk, 95 $\%$ CI = 95 \% confidence interval, Ref = reference category. 
Table 3. Positive bacteria cultures, presence of Staphylococcus aureus in positive bacteria cultures from the tip of the pins and presence of loose pins at removal of 106 patients operated on by the HCO.

$\begin{array}{llll}\begin{array}{l}\text { Group 1 } \\ \mathrm{N}=60\end{array} & \begin{array}{l}\text { Group 2 } \\ \mathrm{n}=46\end{array} & \text { RR (95 \% CI) } \\ & & \text { Group 1 1.0 (Ref) } & \text { p value } \\ \end{array}$

\begin{tabular}{lcccc} 
Pos bact cultures & $22 / 59$ & $19 / 46$ & $1.1(0.6-1.9)$ & ns \\
Staph aureus* & $14 / 59$ & $12 / 46$ & $1.1(0.5-2.3)$ & ns \\
Loose pins & $9 / 59$ & $8 / 46$ & $1.1(0.4-3.0)$ & ns \\
\hline
\end{tabular}

*Presence of Staphylococcus aureus in positive bacteria cultures.

Group 1 = 3 days of infection prophylaxis by antibiotics, Group 2 = one dose of infection prophylaxis by antibiotics.

$\mathrm{HCO}=$ hemicallotasis osteotomy, Pos bact cultures $=$ Positive bacteria cultures, $\mathrm{RR}=$ relative risk, 95 \% CI = 95 \% confidence interval, Ref = reference category. 
Table 4 Relationship of risk factors** to positive cultures, positive cultures with Staphylococcus aureus, loose pins at removal and complications of 106 patients operated on y the HCO.

$\begin{array}{llll}\begin{array}{l}\text { Pos } \\ \text { culture* }^{*}\end{array} & \begin{array}{l}\text { Pos } \\ \text { culture* } \\ \text { Staph aureus }\end{array} & \begin{array}{l}\text { Loose pin } \\ \text { at removal }\end{array} & \end{array}$

OR (95\% CI) and significant values

\begin{tabular}{|c|c|c|c|c|}
\hline Gender & & & & \\
\hline Men & 1.0 (Ref.) & 1.0 (Ref.) & )1.0 (Ref.) & 1.0 (Ref.) \\
\hline Women & $0.6(0.2-1.4)$ & $0.7(0.2-2)$ & $1.3(0.4-4.7)$ & $1.7(0.6-5.1)$ \\
\hline Age & & & & \\
\hline$<50$ & 1.0 (Ref.) & 1.0 (Ref.) & 1.0 (Ref.) & 1.0 (Ref.) \\
\hline $50-59$ & $1.8(0.6-5.3)$ & $1.3(0.4-4.3)$ & )3.4(0.7-17.9) & $1.6(0.5-5.6)$ \\
\hline $60+$ & $1.0(0.3-3.6)$ & $0.2(0.03-1.3)$ & $1.8(0.2-15.8)$ & $1.0(0.2-5.4)$ \\
\hline BMI & & & & \\
\hline$<25$ & 1.0 (Ref.) & 1.0 (Ref.) & 1.0 (Ref.) & 1.0 (Ref.) \\
\hline $25-29$ & $2.3(0.7-7.7)$ & $1.0(0.2-3.9)$ & $0.7(0.1-4)$ & $0.5(0.1-2.2)$ \\
\hline $30+$ & $1.4(0.4-4.5)$ & $1.0(0.3-4.2)$ & $1.7(0.3-8.1)$ & $1.0(0.3-4.1)$ \\
\hline Inf prophylax & & & & \\
\hline 3 days & 1.0 (Ref.) & 1.0 (Ref.) & 1.0 (Ref.) & 1.0 (Ref.) \\
\hline one dose & $0.5(0.2-1.3)$ & $1.6(0.5-4.7)$ & $0.8(0.2-2.8)$ & $1.0(0.3-4.1)$ \\
\hline Hospital & & & & \\
\hline Stay & & & & \\
\hline 1 day & 1.0 (Ref.) & 1.0 (Ref.) & 1.0 (Ref.) & 1.0 (Ref.) \\
\hline$\geq 2$ days & $0.7(0.3-1.8)$ & $0.2(0.08-0.8)$ & $1.9(0.5-6.9)$ & $4.4(1.4-14.3)$ \\
\hline Smoking & & & & \\
\hline Non-smoker & 1.0 (Ref.) & 1.0 (Ref.) & 1.0 (Ref.) & 1.0 (Ref.) \\
\hline Smoker & $0.6(0.2-1.8)$ & $0.2(0.05-0.8)$ & $0.6(0.1-3.1)$ & $4.5(1.3-15.1)$ \\
\hline $\begin{array}{l}\text { Time of } \\
\text { Inf prophylax }\end{array}$ & & & & \\
\hline all other time & 1.0 (Ref.) & 1.0 (Ref.) & 1.0 (Ref.) & 1.0 (Ref.) \\
\hline$\leq 60-\geq 1 \mathrm{~min}$ & $0.8(0.3-2.2)$ & $1.3(0.4-4.2)$ & $0.4(0.1-1.9)$ & $0.7(0.2-2.5)$ \\
\hline
\end{tabular}

$*$ Pos culture $=$ Positive culture at week one and/or six and/or ten.

**Adjusted simultaneously for all other risk factors listed.

***Time of administration of infection prophylaxis.

$\mathrm{HCO}=$ hemicallotasis osteotomy, Staph aureus = Staphylococcus aureus, BMI = body mass index,

Inf prophylaxis $=$ infection prophylaxis, all other time $=$ infection prophylaxis given $>61$ minutes preoperatively, postoperatively or not at all, OR = odds ratio, $95 \% \mathrm{CI}=95 \%$ confidence interval, Ref. = reference category. 